\title{
QUANTIFICAÇÃO DO PESTICIDA DICLORVOS POR VOLTAMETRIA DE ONDA QUADRADA EM ÁGUAS PURAS E NATURAIS
}

Robson T. S. Oliveira e Sergio A. S. Machado*

Departamento de Físico-Química, Instituto de Química de São Carlos, Universidade de São Paulo, CP 780, 13560-970 São Carlos - SP

Recebido em 4/12/03; aceito em 17/5/04; publicado na web em 19/07/04

QUANTIFICATION OF THE PESTICIDE DICHLORVOS BY SQUARE WAVE VOLTAMMETRY IN PURE AND NATURAL WATERS. This work reports the use of square wave voltammetry (SWV) to analyse the electrochemical reduction of dichlorvos (2, 2-dichlorovinyl-dimethylphosphate) in spiked pure and natural waters. SWV measurements were carried out in $0.5 \mathrm{~mol} \mathrm{~L}^{-1}$ $\mathrm{Na}_{2} \mathrm{SO}_{4}$ aqueous solutions at $\mathrm{pH} 5$, prepared with water originated from three different sources, namely, one sample of purified water and others from two urban creeks in São Carlos County. In all cases, two reduction peaks were observed, at potentials of 0.15 and $-1.05 \mathrm{~V}$ vs $\mathrm{Ag} / \mathrm{AgCl}$, with both current and potential being dependent on pesticide concentration. This allowed the calculation of the following detection limits: 1.0, 2.5 and 3.0x10-8 $\mathrm{mol} \mathrm{L}^{-1}$ for purified, Gregorio creek and Monjolinho creek waters, respectively, in a working range between $2.0 \times 10^{-7}$ and $1.4 \times 10^{-6} \mathrm{~mol} \mathrm{~L}^{-1}$. Recovery measurements found values higher than $80 \%$ in all cases, for an added concentration of $4.0 \times 10^{-7} \mathrm{~mol} \mathrm{~L}^{-1}$ of dichlorvos in each solution. All analytical experiments were performed in triplicate and showed a standard deviation always less than $3 \%$.

Keywords: dichlorvos; electroanalysis; pesticides.

\section{INTRODUÇÃO}

O uso intensivo de pesticidas na agricultura tem causado problemas de contaminação do ambiente, assim como de alimentos, águas e ar, bem conhecidos ${ }^{1}$. Desta forma, o monitoramento constante destas espécies, assim como dos seus produtos de degradação, às vezes mais tóxicos que a substância de origem, nas diferentes matrizes, é muito recomendado, justificando os esforços realizados no sentido do desenvolvimento de técnicas e métodos analíticos que visem a melhoria na rapidez e sensibilidade na determinação de pesticidas nestas matrizes ${ }^{2-5}$, assim como explorem a possibilidade de aplicação in situ no campo.

Com referência à aplicabilidade das análises in situ, as técnicas eletroanalíticas (voltamétricas) podem ser de grande valia, pois os sistemas eletroquímicos têm sido miniaturizados e aplicados em pesquisas de campo. Para isto, é necessário, inicialmente, garantir que as moléculas do pesticida em estudo sejam eletroativas (ou seja, sofram um processo de redução ou de oxidação) sobre um eletrodo conveniente, como o eletrodo gotejante de mercúrio ou alguns eletrodos sólidos como platina, ouro, carbono, etc... Um grande número de trabalhos publicados atestam esta eletroatividade de diferentes moléculas de pesticidas sólidos ${ }^{5-12}$.

Assim, pode-se explorar os potenciais destas técnicas voltamétricas em diferentes aplicações, como no estudo da adsorção, degradação e transporte de pesticidas no ambiente ${ }^{13-16}$, na quantificação destes poluentes em amostras ambientais e mesmo em estudos básicos visando a determinação dos mecanismos de redução ou oxidação eletroquímica destas moléculas. Nestes casos, a rapidez da análise, a sensibilidade e a seletividade das técnicas eletroanalíticas são parâmetros de grande importância e que devem ser otimizados antes da aplicação da técnica proposta.

Uma das mais importantes classes de pesticidas são os organosfosforados. Estes são freqüentemente utilizados no combate

*e-mail: sasmach@iqsc.usp.br a insetos que atacam frutos e hortaliças de modo geral ${ }^{17,18}$. Sua intensa utilização, inclusive em campanhas municipais de controles de insetos, como o caso do mosquito da dengue, faz com que esta classe de pesticida esteja envolvida em grande parte dos casos de envenenamento ${ }^{19}$, devido a seus efeitos nocivos à saúde como, por exemplo, efeitos teratogênicos ${ }^{20}$.

O diclorvos, fosfato de 0,0-dimetil-2,2-diclorovinila, cuja fórmula estrutural é apresentada na Figura 1, é um líquido de coloração âmbar, odor aromático, parcialmente solúvel em água $\left(8 \mathrm{mg} \mathrm{L}^{-1} \mathrm{a}\right.$ $25^{\circ} \mathrm{C}$ ) e muito solúvel em hidrocarbonetos aromáticos. A molécula do diclorvos é estável ao aquecimento e é hidrolisada rapidamente em meio alcalino. Entretanto, em meios neutros e ácidos, sua hidrólise é muito lenta ${ }^{5}$. Possui um efeito toxicológico leve em seres humanos, sendo que, em altas quantidades, ataca o sistema nervoso central. O principal problema relacionado à utilização deste pesticida é que o emprego exagerado, tanto doméstico em aerossóis, no combate a moscas, baratas, pernilongos, como na agricultura e pecuária (principalmente no combate a carrapatos em animais confinados), faz com que a contaminação humana se torne muito freqüente.<smiles>COP(=O)(OC)OC=C(Cl)Cl</smiles>

Figura 1. Fórmula estrutural da molécula do pesticida diclorvos (fosfato de 0,0-dimetil-2,2-diclorovinila)

Atualmente, o diclorvos é analisado por uma variedade de técnicas analíticas, a maioria envolvendo a inibição das atividades de enzimas, como a acetilcolinesterase $\mathrm{e}^{21,22}$ ou organofosforados hidrolase $\mathrm{e}^{23}$, além de técnicas cromatográficas como a cromatografia líquida de alta eficiência ${ }^{24}$ e a cromatografia gasosa ${ }^{25}$. Dentro desta va- 
riedade de técnicas, Wilkins et al. ${ }^{21}$ realizaram a determinação quantitativa de pesticidas organofosforados, entre eles o diclorvos, em solventes orgânicos, pela ténica amperométrica baseada na inibição da ação da acetilcolinesterase com um mediador de elétrons, o azul da Prússia. Os experimentos possibilitaram a determinação de pesticidas em faixas sub-micromolares de concentrações, com um tempo total de análise inferior a $10 \mathrm{~min}$. Karousos et al. ${ }^{22}$ desenvolveram um biossensor em cristal de quartzo de uma microbalança eletroquímica de cristal de quartzo onde, por meio da variação de massa do biossensor, puderam determinar os pesticidas carbaril e diclorvos em concentrações até $1 \mathrm{ppm}$. Schöning et al. ${ }^{23}$ utilizaram um biossensor composto pelas enzimas organofosforados hidrolase e acetilcolinesterase num sistema em fluxo para a determinação dos pesticidas organofosforados, inclusive o diclorvos, em uma faixa de concentração de poucos $\mu \mathrm{M}$. Por outro lado, Huang et al. ${ }^{24}$ utilizaram a cromatografia líquida de alta eficiência com detecção quimioluminescente para a determinação de diclorvos, isocarbophos e metil-parathion de folhas de plantas. Neste caso, as curvas de trabalho mostraram um comportamento linear na faixa de concentrações entre 0,086 e $15 \mu \mathrm{g} \mathrm{mL}{ }^{-1}$. Finalmente, Chen et al. ${ }^{25}$ analisaram diclorvos em amostras de vegetais após uma microextração em fase sólida auxiliada por micro-ondas por cromatografia gasosa utilizando um detector de captura de elétrons. Encontraram um limite de detecção de aproximadamente $1,0 \mu \mathrm{g} \mathrm{L^{-1 }}$, numa faixa linear da curva analítica entre os limites de 5 a $75 \mu \mathrm{g} \mathrm{L}^{-1}$.

Neste sentido, visando desenvolver uma metodologia eletroanalítica para a determinação do pesticida diclorvos ${ }^{26-28}$ em águas puras e naturais, utilizou-se a voltametria de onda quadrada e o eletrodo de gota suspensa de mercúrio em diferentes amostras. As amostras de águas foram contaminadas no laboratório, sendo estas constituídas por água pura e águas naturais de córregos coletadas na região urbana de São Carlos - SP.

\section{PARTE EXPERIMENTAL}

\section{Reagentes}

O padrão de diclorvos utilizado foi cedido pela Novartis S/A, apresentando pureza de 99,98\%. O eletrólito suporte utilizado foi preparado com sulfato de sódio Merck PA e água purificada com o sistema Milli-Q (Millipore Inc.), com resistividade superior a $10 \mathrm{M} \Omega$, sendo o pH ajustado com ácido sulfúrico Merck PA.

\section{Equipamentos}

Todos os experimentos eletroquímicos foram realizados utilizando-se um analisador polarográfico modelo 348 B da EG\&G PARC, acoplado a um eletrodo gotejante de mercúrio modelo 303 da EG\&G PARC.

\section{Metodologias}

Os estudos voltamétricos de onda quadrada do pesticida diclorvos foram realizados utilizando-se soluções aquosas de $\mathrm{Na}_{2} \mathrm{SO}_{4}$ $\left(0,5 \mathrm{~mol} \mathrm{~L}^{-1}\right)$ em $\mathrm{pH} 5$, ajustado com $\mathrm{H}_{2} \mathrm{SO}_{4}$ de uma solução estoque $1 \mathrm{~mol} \mathrm{~L}^{-1}$. As soluções padrão do pesticida foram preparadas com água ultra-pura, assim como com amostras de águas naturais coletadas nos Córregos do Monjolinho e do Gregório, da cidade de São CarlosSP. As amostras foram coletadas antes e após estes cursos d'água atravessarem a cidade, em pontos com médio e alto níveis de contaminação por dejetos orgânicos oriundos de esgotos residenciais e industriais, respectivamente. As amostras de água dos córregos foram coletadas em frascos de vidro âmbar, de $1 \mathrm{~L}$, originalmente des- tinados ao acondicionamento de éter de petróleo, evitando, assim, sua contaminação, dada a baixa temperatura de evaporação do éter de petróleo. Os frascos foram enxaguados com água corrente de torneira e tratados com uma solução sulfonítrica $\left(\mathrm{HNO}_{3}: \mathrm{H}_{2} \mathrm{SO}_{4} 1: 1 \mathrm{v} / \mathrm{v}\right)$, enxaguados com água purificada e secos em estufa a $120{ }^{\circ} \mathrm{C}$. As amostras coletadas foram armazenadas em refrigerador, a $4{ }^{\circ} \mathrm{C}$, sendo utilizadas o mais rapidamente possível para evitar qualquer modificação das suas caracterísitcas físico-químicas. Para o procedimento analítico, alíquotas destas amostras tinham o seu $\mathrm{pH}$ ajustados para $5 \mathrm{em} \mathrm{Na} \mathrm{SO}_{4} \cdot 10 \mathrm{H}_{2} \mathrm{O} 0,5 \mathrm{~mol} \mathrm{~L}^{-1}$. As soluções estoque do pesticida eram preparadas neste eletrólito e acrescentadas, no volume apropriado, à célula eletroquímica, contendo eletrólito preparado com água pura.

As soluções analisadas foram desoxigenadas borbulhando-se $\mathrm{N}_{2}$ SS, pré-saturado com o eletrólito, da White Martins, durante $10 \mathrm{~min}$ em uma célula polarográfica de $10 \mathrm{~mL}$.

\section{RESULTADOS E DISCUSSÃO}

\section{Otimização dos parâmetros voltamétricos}

O diclorvos apresentou dois picos de redução em meio aquoso, em potenciais de $-0,15$ e $-1,05 \mathrm{~V}$, para uma concentração de $7,0 \times 10^{-7} \mathrm{~mol} \mathrm{~L}^{-1} \mathrm{em} \mathrm{Na}_{2} \mathrm{SO}_{4} 0,5 \mathrm{~mol} \mathrm{~L}^{-1}, \mathrm{pH} 5,0$, com $\Delta \boldsymbol{E}_{\mathrm{s}}=2 \mathrm{mV}, \boldsymbol{a}$ $=50 \mathrm{mV}, \boldsymbol{f}=80 \mathrm{~Hz}$. Estes valores dos parâmetros voltamétricos foram previamente selecionados como aqueles onde o maior sinal de corrente de pico foi obtido ${ }^{24}$. Ambos os picos apresentaram componentes de correntes direta e reversa, indicando que a reação eletródica é um processo reversível, como observado na Figura 2. Neste caso, a corrente direta é coletada durante a aplicação do pulso de potenciais no sentido da varredura e a corrente inversa, quando o pulso de potenciais é aplicado no sentido contrário ao da varredura. Para se aumentar o sinal analítico, faz-se a soma dos módulos das correntes e a corrente resultante é calculada. Neste trabalho utilizouse a corrente resultante como sinal analítico.

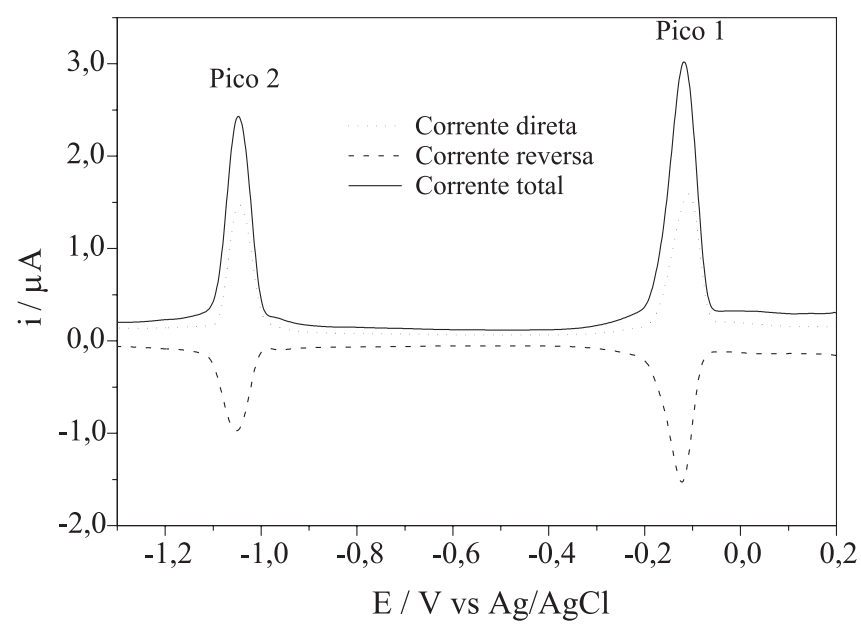

Figura 2. Voltamograma de onda quadrada obtido com o eletrodo de gota

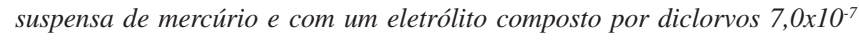
$\mathrm{mol} \mathrm{L}^{-1}$ em $\mathrm{Na}_{2} \mathrm{SO}_{4} 0,5 \mathrm{~mol} \mathrm{~L}^{-1}$ (pH 5,0) com $\Delta E_{s}=2 \mathrm{mV}, a=50 \mathrm{mV}, f=80$ $H z$. As componentes direta, reversa e total da corrente estão indicadas na figura

Resultados prévios ${ }^{5}$ mostraram que, provavelmente, o pico 1 (em $-0,15 \mathrm{~V})$ está relacionado com a redução da dupla ligação da molécula do diclorvos $(\mathrm{C}=\mathrm{C})$, enquanto que o pico $2(\mathrm{em}-1,05 \mathrm{~V})$ referese a uma declorificação da molécula. Entretanto, para uma associa- 
ção definitiva, novos estudos estão sendo feitos com análises de produtos de eletrólise e os resultados serão tema de publicação futura.

A variação da concentração hidrogeniônica do meio (valores de pH entre 2,5 e 8,5) não mostrou qualquer influência, tanto nos potenciais como nas correntes de pico, de ambos os picos 1 e 2. Esta independência dos potenciais de pico indica que a redução da molécula do diclorvos, nas condições dos experimentos, não é precedida por processos de protonação, o que é esperado devido à ausência de grupos protonáveis na molécula do pesticida.

$\mathrm{O}$ parâmetro equivalente à velocidade de varredura da voltametria cíclica, na voltametria de onda quadrada (VOQ), é a frequiência de aplicação dos pulsos, $f$. A otimização deste parâmetro é de fundamental importância pois, para a maioria dos processos eletródicos, a corrente de pico é diretamente proporcional ao seu valor. Assim, com a maximização do valor de $f$, aumenta-se, conseqüentemente, a sensibilidade da técnica experimental. A Figura 3 mostra a relação linear entre as correntes de pico e a frequiência, para os picos 1 e 2 de redução do diclorvos, no intervalo entre 10 e $120 \mathrm{~Hz}$. O maior valor de freqüência foi definido pelas limitações do potenciostato utilizado que, devido ao seu tempo de resposta, não permite a coleta de dados de corrente com um intervalo de tempo menor. Observa-se que, para $f=0$, a corrente de pico não é zero, pois, nestas condições, o experimento torna-se uma voltametria de varredura linear, com a corrente de pico equivalente à obtida por aquela técnica não pulsada. Assim, nas condições experimentais deste trabalho, optou-se pela freqüência de $100 \mathrm{~Hz}$, como o valor ótimo para as análises.

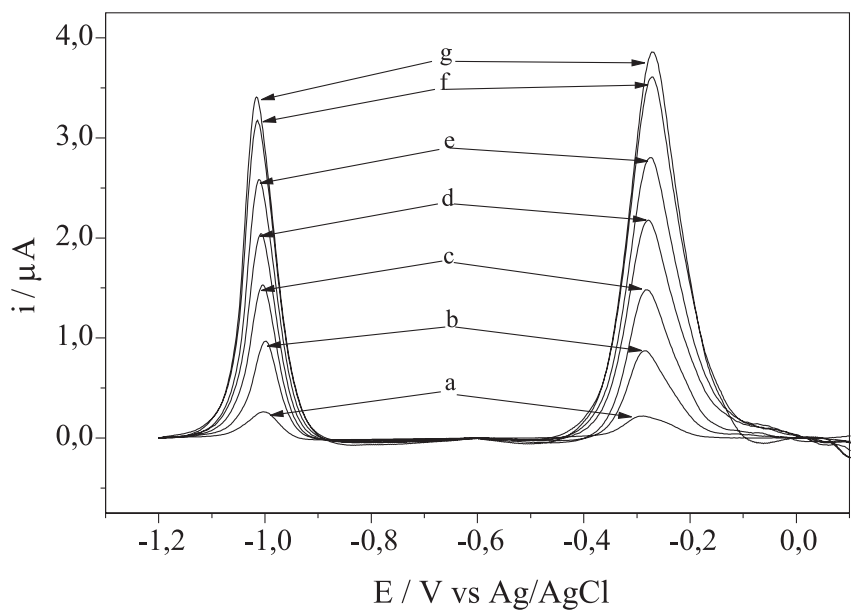

Figura 3. Efeito da variação da freqüência de aplicação dos pulsos de potenciais da onda quadrada nos perfis voltamétricos obtidos para uma solução de diclorvos $7,0 \times 10^{-7} \mathrm{~mol} \mathrm{~L}^{-1}$ em $\mathrm{Na}_{2} \mathrm{SO}_{4} \cdot 10 \mathrm{H}_{2} \mathrm{O} 0,5 \mathrm{~mol} \mathrm{~L}^{-1}$ ( $\mathrm{pH}$ 5,0) com $\Delta E_{s}=2 \mathrm{mV}, a=50 \mathrm{mV}, f=10(\mathrm{a}), 30(\mathrm{~b}), 50(\mathrm{c}), 70(\mathrm{~d}), 90(\mathrm{e}), 110$ (f) e $120(\mathrm{~g}) \mathrm{Hz}$

Outro parâmetro passível de otimização, na técnica da voltametria de onda quadrada é a amplitude do pulso de potencial aplicado, $a$. A Figura 4 mostra o efeito da variação deste parâmetro, entre 10 e 120 $\mathrm{mV}$, nas correntes de pico. A relação apresenta um comportamento linear até $50 \mathrm{mV}$, o que está de acordo com o esperado, na teoria, para processos reversíveis com adsorção de reagentes ou produtos ${ }^{29}$. Assim, escolheu-se o valor de $50 \mathrm{mV}$ para utilização posterior nas análises.

Finalmente, a variação do incremento de potencial da rampa, $\Delta E_{s}$. não causou um efeito marcante nos potenciais e correntes de pico dos voltamogramas de onda quadrada para a redução do diclorvos sobre eletrodo de gota suspensa de mercúrio. Assim, o valor de $2 \mathrm{mV}$, foi escolhido para este trabalho.

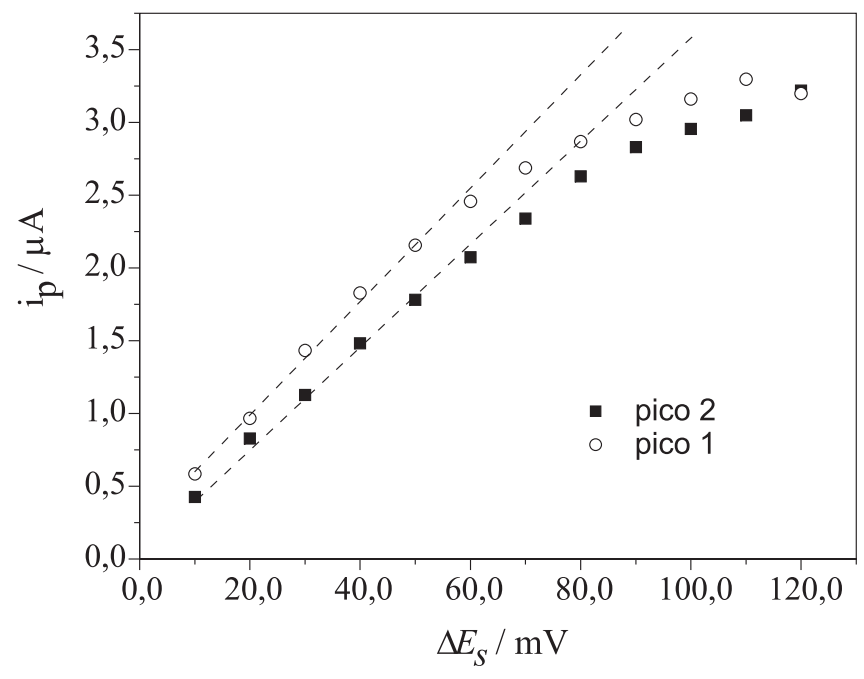

Figura 4. Efeito da variação da amplitude dos pulsos de potenciais utilizados na onda quadrada nas correntes de pico obtidas dos voltamogramas de onda quadrada para a redução do diclorvos $7,0 \times 10^{-7} \mathrm{~mol} \mathrm{~L} \mathrm{~L}^{-1} \mathrm{em}$ $\mathrm{Na}_{2} \mathrm{SO}_{4} \cdot 10 \mathrm{H}_{2} \mathrm{O} 0,5 \mathrm{~mol} \mathrm{~L}^{-1}\left(\mathrm{pH} \mathrm{5,0)} \mathrm{com} \Delta E_{s}=2 \mathrm{mV}, f=100 \mathrm{~Hz}\right.$

\section{Procedimentos analíticos}

A dependência linear das correntes de pico em função da concentração do pesticida adicionado ao eletrólito, para ambos processos eletródicos, sugere a sua utilização para fins analíticos. Entretanto, o pico em -0,15 V mostrou-se mais sensível à presença de impurezas presentes no eletrólito e, assim, escolheu-se o pico catódico em -1,05 V para os fins analíticos deste trabalho.

As respostas voltamétricas de onda quadrada para a redução do diclorvos como função da concentração adicionada do pesticida estão apresentadas na Figura 5. As correntes de pico obtidas destes voltamogramas, representadas em função das concentrações adicionadas do pesticida, resultam numa curva analítica com equação da reta igual a $\mathrm{i}=-124,7+4,80 \times 10^{3} \mathrm{~A} \mathrm{~L} \mathrm{~mol}^{-1}, \mathrm{r}^{2}=0,9985$, desvio

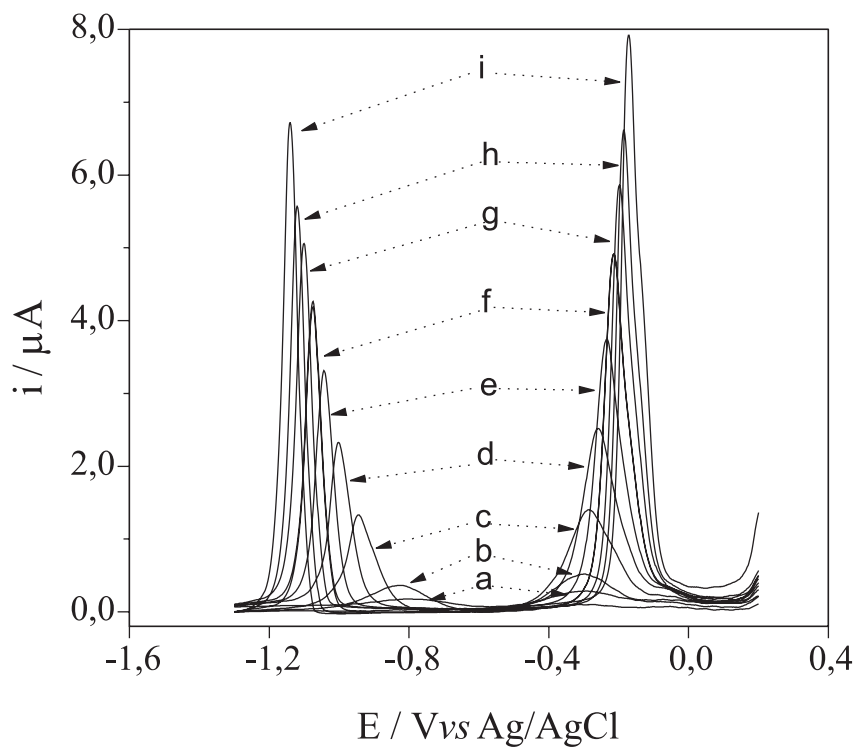

Figura 5. Voltamogramas de onda quadrada para diferentes concentrações adicionadas de diclorvos em $\mathrm{Na}_{2} \mathrm{SO}_{4} 10 \mathrm{H}_{2} \mathrm{O} 0,5 \mathrm{~mol} \mathrm{~L}^{-1}$ (pH 5,0) (preparado com água pura) com $\Delta E_{s}=2 \mathrm{mV}, a=50 \mathrm{mV}, f=100 \mathrm{~Hz}$. Concentrações de diclorvos: $1,0 \times 10^{-7}(a) ; 2,0 \times 10^{-7}(b) ; 3,8 \times 10^{-7}(c) ; 5,6 \times 10^{-7}(d) ; 7,4 \times 10^{-7}(e)$;

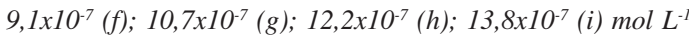


padrão $=131$, número de pontos $=10$, com um intervalo de linearidade entre $2,0 \times 10^{-8}$ a $1,46 \times 10^{-6} \mathrm{~mol} \mathrm{~L}^{-1}$.

A Figura 6 mostra as respostas voltamétricas para alíquotas do eletrólito contendo concentrações conhecidas do diclorvos, preparadas com água coletada no Córrego do Monjolinho, em função da concentração adicionada do pesticida. Um perfil análogo é encontrado para as respostas voltamétricas com água do Córrego do Gregório. A partir das respectivas correntes de pico foram construídas as curvas analíticas que estão apresentadas na Figura 7. Pode-se observar que as respectivas tangentes das curvas analíticas (que retratam a sensibilidade da técnica) apresentaram valores menores que aquele obtido com o eletrólito preparado com água pura, o que pode ser associado com a presença de interferentes na matriz das águas dos córregos. Em trabalhos anteriores ${ }^{2-4}$ foi mostrado que, mesmo com água coletada em córregos com baixos teores de poluentes domésticos ou industriais, se observa uma sensível diminuição na sensibilidade em relação à água pura. Esta diminuição da sensibilidade foi atribuída à presença de ácidos húmicos e fúlvicos. Estas

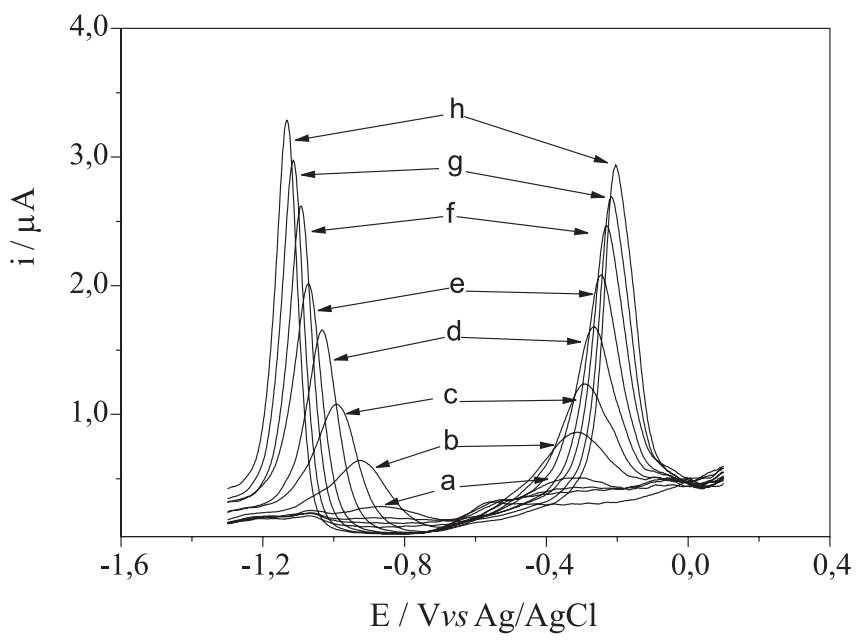

Figura 6. Voltamogramas de onda quadrada para a redução do diclorvos em diferentes concentrações do pesticida em $\mathrm{Na}_{2} \mathrm{SO}_{4} 10 \mathrm{H}_{2} \mathrm{O} 0,5 \mathrm{~mol} \mathrm{~L}^{-1}$ ( $\mathrm{pH}$ 5,0) (preparado com água coletada no Córrego Monjolinho) e condições experimentais idênticas às da Figura 5

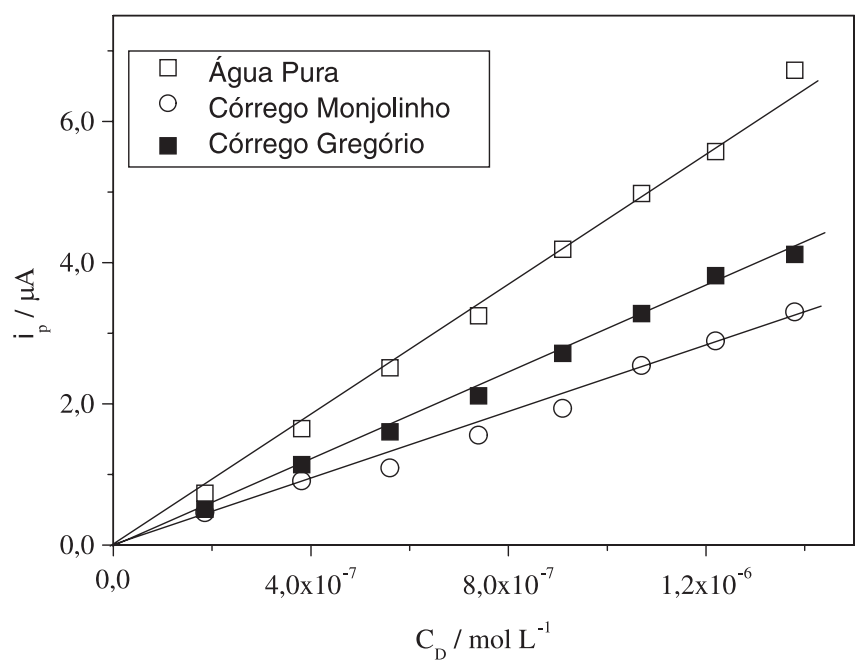

Figura 7. Curvas analíticas obtidas das relações entre as correntes de pico e as concentrações de diclorvos mostradas nas Figuras 5 e 6 . Os resultados estão dentro de um intervalo de confiança de $95 \%$ substâncias podem interferir de duas maneiras distintas: (a) adsorvendo-se na superfície do eletrodo, diminuem a área eletroativa disponível e, portanto, as correntes de pico observadas e (b) podem complexar as moléculas de pesticida, tornando-as inacessíveis para a reação eletródica, ao menos na região de potenciais estudada.

Os resultados obtidos das curvas analíticas estão coletados na Tabela 1.

Tabela 1. Resultados de regressão linear das curvas analíticas do diclorvos, obtidas para as águas puras e naturais

\begin{tabular}{lccc}
\hline Água & $\mathrm{A}\left(10^{11} \mathrm{~A}\right)$ & $\mathrm{B}\left(\mathrm{A} \mathrm{L} \mathrm{mol}^{-1}\right)$ & $\mathrm{R}$ \\
\hline Pura & $-1,2 \pm 0,5$ & $4,8 \pm 0,1$ & 0,998 \\
Córrego Gregório & $-3,1 \pm 0,6$ & $3,29 \pm 0,07$ & 0,998 \\
Córrego Monjolinho & $-2,9 \pm 0,6$ & $2,56 \pm 0,07$ & 0,997 \\
\hline
\end{tabular}

\section{Determinação dos limites de detecção e quantificação}

Os limites de detecção e de quantificação (LD e LQ, respectivamente) são características do desempenho de cada procedimento de análise e dependem de certas características do processo em estudo. Segundo a IUPAC ${ }^{30}$, estes parâmetros podem ser calculados utilizando-se as equações

$\mathrm{LD}=3 \mathrm{Sb} / \mathrm{B}$ e $\mathrm{LQ}=10 \mathrm{Sb} / \mathrm{B}$

Nestas equações, Sb corresponde ao valor do desvio padrão dos valores de corrente obtidos da curva relacionada ao branco, no potencial relativo ao potencial de pico para a redução do diclorvos. Assim, 10 voltamogramas de onda quadrada foram obtidos para o branco e os 10 valores de corrente, em $-1,05 \mathrm{~V}$, foram analisados, produzindo um valor médio e um desvio padrão, sendo este último utilizado na equação (1). Por outro lado, B é o valor da tangente das curvas de trabalho para as diferentes matrizes, como apresentado na Tabela 1.

Os valores dos desvios padrão dos brancos e dos limites de detecção e quantificação obtidos para as diferentes amostras analisadas estão apresentados na Tabela 2.

Tabela 2. Valores obtidos dos limites de detecção e quantificação para o diclorvos nas águas puras e naturais

\begin{tabular}{lccc}
\hline Água & $\mathrm{S}_{\mathrm{b}}$ & $\begin{array}{c}\mathrm{LD} \\
\left(10^{8} \mathrm{~mol} \mathrm{~L}^{-1}\right)\end{array}$ & $\begin{array}{c}\mathrm{LQ} \\
\left(10^{8} \mathrm{~mol} \mathrm{~L}^{-1}\right)\end{array}$ \\
\hline Pura & 16,9 & 1,1 & 3,6 \\
Córrego Gregório & 32,9 & 2,5 & 8,3 \\
Córrego Monjolinho & 21,4 & 3,0 & 9,9 \\
\hline
\end{tabular}

O aumento nos valores dos limites de detecção observados está associado à crescente quantidade de substâncias orgânicas contidas nas amostras naturais (inclusive os poluentes domésticos e industriais), de acordo com a discussão do item anterior.

Entretanto, os valores calculados estão, em todos os casos, bem abaixo daqueles valores máximos permitidos pelo CONAMA (Conselho Nacional do Meio Ambiente) para pesticidas organofosforados em águas naturais, ou seja, de $4,5 \times 10^{-7} \mathrm{~mol} \mathrm{~L}^{-131}$. Desta forma, esta metodologia pode ser empregada para se testar a contaminação ou não de águas, mesmo intensamente poluídas, como as dos córregos aqui analisados, quanto à presença do pesticida diclorvos.

\section{Experimentos de recuperação}

Os experimentos de recuperação foram feitos utilizando-se as 
curvas analíticas apresentadas no item acima. Para estes experimentos, utilizou-se o método da adição de padrão.

Para a determinação voltamétrica do diclorvos, foram adicionados $400 \mathrm{~mL}$ de uma solução estoque do diclorvos $\left(1,0 \times 10^{-5} \mathrm{~mol} \mathrm{~L}^{-1}\right)$ em $10 \mathrm{~mL}$ de eletrólito de suporte (água pura ou natural em $\mathrm{Na}_{2} \mathrm{SO}_{4}$

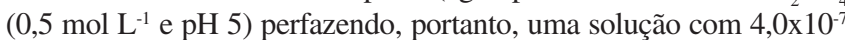
mol L $\mathrm{L}^{-1}$ do pesticida. Foram realizadas três determinações para cada tipo de amostra e os resultados referentes aos testes de recuperação podem ser visualizados na Tabela 3. Pode-se observar uma diminuição nos valores de recuperação das amostras naturais em relação aos valores obtidos para amostras de águas puras. Isso se deve principalmente ao efeito dos altos níveis de interferentes presentes nas amostras naturais, como já mencionado anteriormente. Entretanto, devese levar em conta os baixos valores de concentração utilizados para a realização dos testes de recuperação, pois estes estão em níveis de traços justificando, desta forma, os desvios observados dos resultados experimentais em relação ao valor teórico de $100 \%$.

Tabela 3. Resultados dos experimentos de recuperação para o diclorvos, obtidas em amostras de águas puras e naturais

\begin{tabular}{lccc}
\hline Água & $\begin{array}{c}\text { Quantidade } \\
\text { adicionada } \\
\left(10^{7} \mathrm{~mol} \mathrm{~L}^{-1}\right)\end{array}$ & $\begin{array}{c}\text { Quantidade } \\
\text { encontrada } \\
\left(10^{7} \mathrm{~mol} \mathrm{~L}^{-1}\right)\end{array}$ & $\begin{array}{c}\text { Recuperação } \\
\text { média } \\
(\%)\end{array}$ \\
\hline Pura & 4,0 & $3,7 \pm 0,1$ & 93,0 \\
Córrego Gregório & 4,0 & $3,3 \pm 1$ & 82,5 \\
Córrego Monjolinho & 4,0 & $3,3 \pm 0,8$ & 82,5 \\
\hline
\end{tabular}

\section{CONCLUSÕES}

No presente trabalho aplicou-se a voltametria de onda quadrada com sucesso em um procedimento eletroanalítico para a quantificação do pesticida diclorvos em água pura e também em amostras obtidas de córregos urbanos poluídos. A técnica voltamétrica utilizada apresentou bons resultados no que diz respeito à sensibilidade e ao tempo de análise. Além disso, foi possível realizar testes de recuperação com excelente reprodutibilidade. Desta forma, torna-se possível o desenvolvimento de uma metodologia analítica alternativa para a quantificação do diclorvos em diversos tipos de matrizes aquáticas.

Após a obtenção das curvas analíticas, observou-se que os limites de detecção e quantificação aumentaram, na transposição da metodologia da água pura, de laboratório, para as amostras naturais, provavelmente devido ao aumento dos níveis de interferentes. Contudo, os valores encontrados são baixos o suficiente para permitir uma análise a nível de traços de diclorvos, nestas situações, atingindo limites de detecção até da ordem de $10^{-8} \mathrm{~mol} \mathrm{~L}^{-1}$, sem a necessidade de etapas prévias separações, 'clean-up' ou preconcentrações, diminuindo assim o custo e o tempo das análises.

\section{AGRADECIMENTOS}

Os autores agradecem ao CNPq e à FAPESP (Proc. No. 2001/ 13358-3) pelos auxílios concedidos.

\section{REFERÊNCIAS}

1. O Estado de São Paulo de 30/09/1998 e de 01/10/1998.

2. Vaz, C. M. P.; Crestana, S.; Machado, S. A. S.; Mazo, L. H.; Massaroppi, M. R. C.; Avaca, L. A.; Pesticidas Revista Técnico Científica 1996, 6, 55.

3. Vaz, C. M. P.; Crestana, S.; Machado, S. A. S.; Mazo, L. H.; Avaca, L. A.; Int. J. Environ. Anal. Chem. 1996, 62, 65.

4. Vaz, C. M. P.; Crestana, S.; Machado, S. A. S.; Mazo, L. H.; Avaca, L. A.; Electroanalysis 1997, 9, 475.

5. Oliveira, R. T. S.; Dissertação de Mestrado, Instituto de Química de São Carlos, Universidade de São Paulo, 2000.

6. Lippolis, M. T.; Concialini, V.; Talanta 1988, 35, 235.

7. Bourque, C. L.; Duguai, M. M.; Gautreau, Z. M.; Int. J. Anal. Chem. 1989, $37,187$.

8. Lechien, A.; Valenta, P.; Nurnbergh, H. W.; Patriarche, G. J.; Fresenius J. Anal. Chem. 1981, 306, 156.

9. Sedeño, P. Y.; Villena, J. M.; Pingarrón, J. M.; Polo, L. M.; Anal. Chim. Acta 1992, 264, 141.

10. Guiberteau, A.; Días, T. G.; Salinas, F.; Ortiz, J. M.; Anal. Chim. Acta 1995, 305, 219.

11. Corbini, G.; Biondi, C.; Proietti, D.; Dreassi, E.; Corti, P.; Analyst 1993, $118,183$.

12. Walcarius, A.; Lamberts L.; J. Electroanal. Chem. 1996, 406, 59.

13. Filimonova, M. M.; Gorbunova, V. E.; Z. Analiticheskoi Khimii 1973, 28, 1184.

14. Miles, J. R.; Harris, C. R.; J. Environ. Sci. Health 1978, 13, 199.

15. Frank, R.; Braun, H. E.; Ishida, K.; Suda, P.; Can. J. Soil Sci. 1976, 56, 463.

16. Harris, C. R.; Sans, W. W.; Pest. Monit. J. 1971, 5, 259.

17. Elzbieta, L.; Sowalskar, O.; Bromotol A. G.; Chem. Toksykol. 1977, 10, 97.

18. Sergeant, D. B.; Zifko, V.; Tech. Rep. Fish. Mar. Serv. (Can.) 1979, 886, 75 .

19. Shimizu, K.; Shiono, H.; Fukushima, T.; Sasaki, M.; Akutzu, H.; Sakata, M.; Forensic Sci. Int. 1996, 83, 61.

20. Temolin, C.; The Pesticide Manual (incorporating the Agrochemicals Handbook), 10 ${ }^{\text {th }}$ ed., Surrey, Crop Protection Publications, 1994, p. 313.

21. Cris, S.; Tarrant, K. R.; Analyst 1971, 96, 310.

22. Wilkins, E.; Carter, M.; Voss, J.; Ivnitski, D.; Electrochem. Commun. 2000, 2, 786 .

23. Karousos, N. G.; Aouabdi, S.; Way, A. S.; Reddy, S. M.; Anal. Chim. Acta 2002, 469, 189.

24. Schöning, M. J.; Krause, R.; Block, K.; Musahmeh, M.; Mulchandani, A.; Wang, J.; Sens. Actuators, B 2003, 95, 291.

25. Huang, G.; Ouyang, J.; Baeyens, W. R. G.; Yang, Y.; Tao, C.; Anal. Chim. Acta 2002, 474, 21.

26. Chen, Y-I.; Su, Y. S.; Jen, J. F.; J. Chromatogr., A 2002, 976, 349.

27. Machin, A. F.; Quick, M. P.; Walddel, D. F.; Analyst 1973, 98, 176.

28. Mattson, A.; Spillane, J.; Pearce, G.; J. Agric. Food Chem. 1955, 3, 319.

29. Lovric, M.; Sebojka, K.; Murray, W.; Electrochim. Acta 1988, 33, 739.

30. Currie, L. A.; Pure Appl. Chem. 1995, 67, 1699.

31. http://www.polmil.sp.gov.br/unidades/cpfm/legis/CONA20_86.doc, acessada em Fevereiro 2004. 